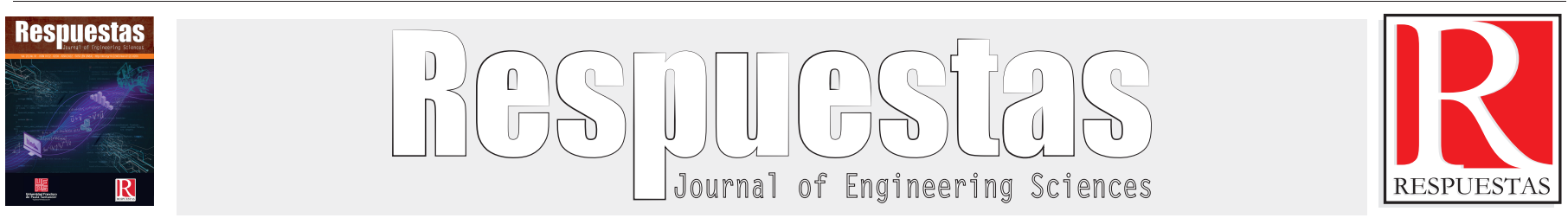

Artículo de Reflexión

https://doi.org/10.22463/0122820X.1507

\title{
Coopetencia, modelo de Gestión Empresarial para Mipymes de Ocaña
}

\section{Coopetition, Business Management model For Ocaña Mipymes}

Maribel Cárdenas-García,"B Blanca Mery Velasco-Burgos, Jorge de Jesús Cañizares-Arévalo.

a Doctora en Educación, mcardenasg@ufpso.edu.co, orcid.org/0000-0003-3404-8806, Universidad Francisco de Paula Santander, Cúcuta, Colombia.

${ }^{b}$ Magister en Gerencia de Empresas, bmvelasco@ufpso.edu.co, orcid.org/0000-0003-1489-0641, Universidad Francisco de Paula Santander Ocaña, Ocaña, Colombia

Magíster en Ciencias Politicas, jjcanizaresa@ufpso.edu.co, orcid.org/0000-0002-7838-4695, Universidad Francisco de Paula Santander Ocaña, Ocaña, Colombia.

Como citar: M. Cárdenas-García, B.M. Velasco-Burgos y J.deJ. Cañizares-Arévalo, "Coopetencia, modelo de Gestión Empresarial para Mipymes de Ocaña", Respuestas, vol. 23, no. S1, pp. 59-65, 2018. https://doi.org/10.22463/0122820X.1507

Recibido: Febrero 5, 2018; Aprobado: Julio 17, 2018

\section{RESUMEN}

\section{Palabras Clave:}

Coopetencia

Desarrollo Sostenible

Globalización Económica

Responsabilidad Social Empresarial

El presente artículo se fundamentó en el diagnóstico situacional efectuado a las micro, pequeñas y medianas empresas (Mipymes) de Ocaña, Norte de Santander, producto de la investigación principal, que tuvo como propósito promover el crecimiento y desarrollo económico de las Mipymes; para lo cual, se conformó una corporación bajo el modelo de gestión empresarial "coopetencia", en aras de lograr la transformación de su accionar bajo la perspectiva social, ambiental y empresarial, desde una mirada de Ocaña como ciudad de negocios, innovadora, inteligente, emprendedora, disminuyendo la huella ecológica que por la operación sin planificación amenaza los recursos naturales y la dignidad laboral. La metodología aplicada se enmarcó bajo el enfoque cuantitativo, con un nivel de investigación descriptiva y exploratoria, un diseño documental y de campo, como instrumento de recolección de información se aplicó la encuesta a 64 Mipymes del municipio. Los principales resultados demostraron que el $98 \%$ de los encuestados desconocen los requisitos legales ambientales aplicables a su actividad económica, donde se evidenció la sinergia entre el empleador-empleado y su relación con el entorno, así mismo, se resaltan las fortalezas encontradas en lo concerniente al proceso administrativo y económico de las Mipymes. Finalmente se vislumbra la necesidad de enfocar y aunar esfuerzos que organicen todas las estrategias competitivas de las Mipymes con perspectivas de responsabilidad social empresarial, ambiental, de seguridad y salud en el trabajo, incorporando factores diferenciadores que les permitan ser sustentables, productivas y competitivas en los diferentes mercados.

\section{ABSTRACT}

Keywords:

Coopetition

Sustainable Development

Economic Globalization

Corporate Social Responsibility

The present article was based on the situational diagnosis effected to the micro, small and medium companies Ocaña (Mipymes), North of Santander, product of the principal investigation, which had as intention promote the growth and economic development of the Mipymes; for which, a corporation conformed under the model of business management "coopetencia", in altars of achieving the transformation of his to gesticulate under the social, environmental and managerial perspective, from Ocaña look as business, innovative, intelligent, enterprising city, diminishing the ecological fingerprint that for the operation without planning threatens the natural resources and the labor dignity. The applied methodology placed under the quantitative approach, with a level of descriptive investigation and exploratory research, a documentary and field design as a tool for information gathering survey to 64 Mipymes the municipality was applied. The principal results showed that $98 \%$ of respondents are unaware of the environmental legal requirements applicable to its economic activity, where the synergy between employer-employee and its relationship with the environment, so it was evident, the strengths found concerning highlights administrative and economic process of Mipymes. Finally the need to focus and combine efforts to organize all the competitive strategies of Mipymes with prospects of business, environmental, safety and health at work social responsibility, incorporating differentiating factors that allow them to be sustainable, productive and competitive in looms different markets.

\section{Introducción}

La globalización ha contribuido a que las grandes y pequeñas empresas redefinan sus estrategias de negociación, para ello las tecnologías de información y comunicación han jugado un papel preponderante para la realización de sus negocios on line. En estos nuevos contextos las organizaciones enfrentan retos cada vez más exigentes en cuanto a los niveles de competitividad, lo que las obliga a ser mucho más eficaces, eficientes y efectivas dentro del sistema económico respondiendo en forma satisfactoria a las expectativas y necesidades del mercado. De otro modo, las que no logren ser competitivas por falta de recursos económicos, físicos, tecnológicos y humanos cualifi- cados tenderán a desaparecer, por lo que deberán aliarse estratégicamente con otras organizaciones que les permita penetrar en forma sostenible en la dinámica apetecida por el sistema capitalista del libre mercado, como lo es la globalización económica.

Frente a estas circunstancias Francés (2006), citado por (Serna, 2014, pág. 43) ha propuesto nuevos paradigmas en estrategias competitivas "coopetencia, designio estratégico, la estrategia del océano azul y la hipercompetencia". Y es la coopetencia el paradigma estratégico escogido como fundamento teórico por parte de los autores como eje central de la investigación y representado en la figura 1 .

*Autor de Correspondencia. 
En relación con el paradigma estratégico “coopetencia”, Porter citado por (Serna, 2014, págs. 32,33), “definió un conjunto de estrategias competitivas que en general podrían agruparse en: (...) De cooperación o alianzas: mediante las cuales varias empresas combinan esfuerzos para competir más eficiente $\mathrm{y}$ eficazmente".

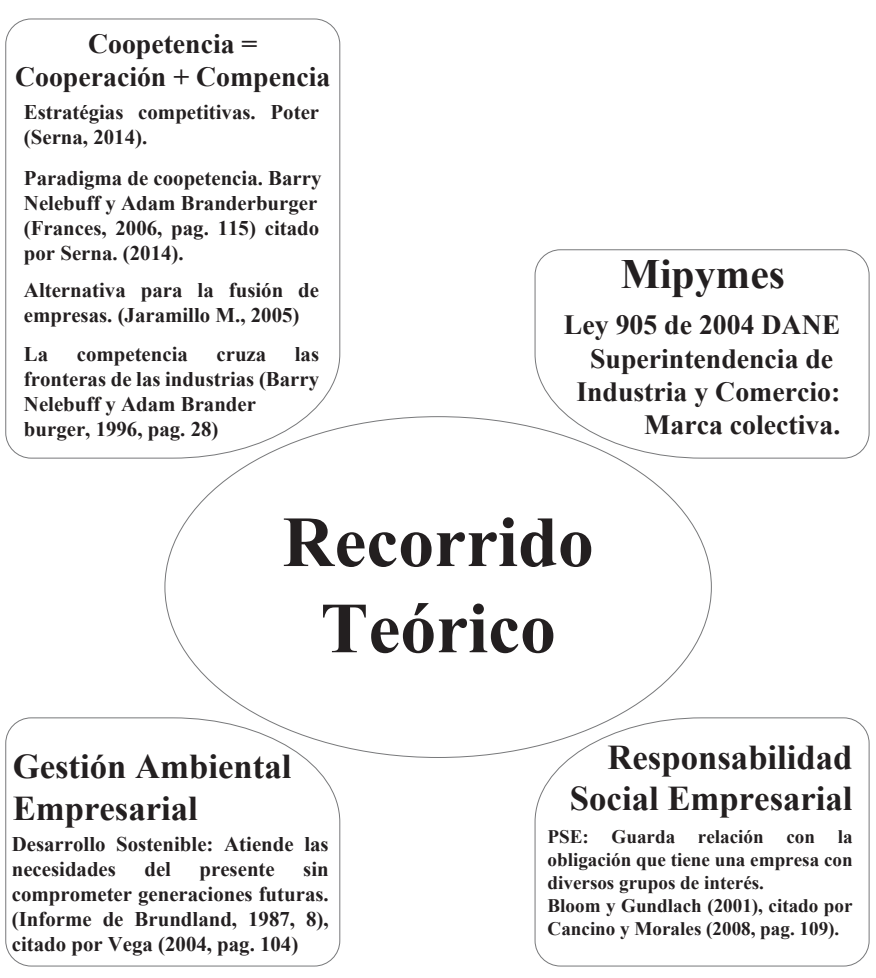

Figura 1. Recorrido Teórico

En este propósito, la cooperación es una estrategia relevante en las organizaciones del siglo XXI para ser competitivas en los diferentes mercados.

Así mismo Serna (2014) ha citado a Porter en lo que respecta al pensamiento estratégico afirmando lo siguiente: "Siguiendo el pensamiento estratégico de Porter, las alternativas estratégicas que una empresa o unidad de negocios enfrenta en una situación coyuntural podrían clasificarse como (...) diversificación, riesgos compartidos, atrincheramiento, innovación y alianzas estratégicas" (p.33).

En lo referente a la coopetencia igualmente (Serna, 2014, pág. 43), cita a Barry Nelebuff y Adam Branderburger (1996) y para ello llegó a afirmar que los autores:

... han presentado un nuevo paradigma que se fundamenta en la cooperación entre los actores del mercado. A esta estrategia se la ha denominado la coopetencia, nombre acuñado por Ray Noorda, fundador de la empresa estadounidense de software Novel. Noorda ha dicho: "Uno tiene que competir y cooperar al mismo tiempo".

De la misma manera, (Jaramillo, 2005, pág. 253) considera que la cooperación es una alternativa para la fusión de empresas, la cual busca: reducir la incertidumbre propia del contexto económico, tornar más confiable el proceso de asimilación de la información, reducir costos, optimizar las ventajas que cada una posee por separado y denomina la coopetencia como cooperación más competencia.

La coopetencia como modelo de cooperación, puede ser considerada como una alternativa de organización para las Mipymes, las cuales presentan dificultades en los procesos de planeación, organización, producción, control, ambiental y de responsabilidad social empresarial, por ello, es fundamental innovar sus estrategias de crecimiento y competencia a través de la unión de esfuerzos colectivos orientados a la competitividad.

Ahora bien, en cuanto a la competencia (Nelebuff \& Brandenburger, 1996, pág. 28) sustentan que "la competencia por conseguir proveedores a menudo cruza las fronteras de las industrias. Los que suministran capital son proveedores, y la competencia por atraer sus fondos se lleva a cabo en un mercado global"'.

Por otra parte, al referirse solo en las pequeñas y medianas empresas (Pymes) éstas conforman el 98\% de las empresas del país, empleando el $50 \%$ de la fuerza laboral nacional (LaNotaEconómica.co, 2010), evidenciándose una importante participación de dicho grupo en la economía del país. Cabe resaltar que en Colombia existe la Asociación Colombiana de las Micro, Medianas y Pequeñas Empresas (ACOPI), encargada de "Dinamizar la economía del país con el fortalecimiento eficiente de las Mipyme..." (ACOPI, 2016).

En este mismo sentido, la legislación Colombiana mediante la (Ley 905, 2004), define:

Artículo $2^{\circ}$ : Para todos los efectos, se entienden por micro incluidas la famiempresas, pequeña y mediana empresa, a toda unidad de explotación económica, realizada por una persona natural o jurídica, en actividades empresariales, agropecuarias, industriales, comerciales o de servicios, rural o urbana, que corresponda a dos de los siguientes parámetros.

Para lo cual en la tabla I se presentan los parámetros establecidos por Ley en lo que corresponde a las Mipymes en Colombia:

Tabla I. Parámetros conforme a la Ley 905 de 2004 para las MIPYMES en Colombia

\begin{tabular}{|c|c|c|c|}
\hline PARAMETRO & MEDIANA EMPRESA & $\begin{array}{c}\text { PEQUEÑA } \\
\text { EMPRESA }\end{array}$ & $\begin{array}{c}\text { MICRO } \\
\text { EMPRESA }\end{array}$ \\
\hline $\begin{array}{c}\text { PLANTA DE } \\
\text { PERSONAL }\end{array}$ & $\begin{array}{c}\text { Entre 51 y 200 } \\
\text { trabajadores }\end{array}$ & $\begin{array}{c}\text { Entre 11 y 50 } \\
\text { trabajadores }\end{array}$ & $\begin{array}{c}\text { Menor o igual a } \\
10 \text { trabajadores }\end{array}$ \\
\hline $\begin{array}{c}\text { ACTIVOS } \\
\text { TOTALES }\end{array}$ & $\begin{array}{c}\text { Entre 5.001 y 30.000 } \\
\text { Smmlv }\end{array}$ & $\begin{array}{c}\text { Entre 501 y } \\
\mathbf{5 . 0 0 0} \text { Smmlv }\end{array}$ & $\begin{array}{c}\text { Menor a 500 } \\
\text { Smmlv, Excluida } \\
\text { la vivienda }\end{array}$ \\
\hline
\end{tabular}

Fuente. Ley 905 de 2004 
Llevando esta información al plano local (municipio de Ocaña), y analizando la base de datos suministrada por la Cámara de Comercio de Ocaña a 30 de abril de 2016 correspondiente a la actualización del registro mercantil de establecimientos de comercio, las Mipymes representan el 100\% del total de los establecimientos comerciales ubicados en la ciudad. La distribución de estas, de acuerdo a la Ley 905 de 2004, se relaciona en la tabla II:

Tabla II. Distribuciones de establecimientos comerciales de acuerdo a la Ley 905 de 2004

\begin{tabular}{|c|c|c|}
\hline MIPYMES & CANTIDAD & PARTICIPACIÒN \\
\hline Microempresas & $\mathbf{5 7 8 2}$ & $\mathbf{9 8 \%}$ \\
\hline Pequeñas Empresas & 109 & $\mathbf{1 . 8 \%}$ \\
\hline Medianas Empresas & 12 & $\mathbf{0 , 2} \%$ \\
\hline Grandes Empresas & $\mathbf{0}$ & $\mathbf{0 \%}$ \\
\hline TOTAL & $\mathbf{5 9 0 5}$ & $\mathbf{1 0 0 \%}$ \\
\hline
\end{tabular}

Con respecto a la actividad económica más representativa y dinamizadora del mercado ocañero es el COMERCIO, tal como se detalla en la tabla III:

Tabla III. actividad económica más representativa y dinamizadora del mercado ocañero

\begin{tabular}{|c|c|}
\hline ACTIVIDAD ECONOMICA & NUMERO \\
\hline $\begin{array}{c}\text { Comercio al por menor de todo tipo de calzado y } \\
\text { artículos de cuero y sucedáneos del cuero en } \\
\text { estable }\end{array}$ & 108 \\
\hline $\begin{array}{c}\text { Mantenimiento y reparación de vehículos } \\
\text { automotores }\end{array}$ & 110 \\
\hline $\begin{array}{c}\text { Comercio al por menor de otros artículos } \\
\text { domésticos en establecimientos especializados }\end{array}$ & 112 \\
\hline $\begin{array}{c}\text { Comercio al por menor de combustible para } \\
\text { automotores } \\
\end{array}$ & 134 \\
\hline $\begin{array}{c}\text { Expendio de bebidas alcohólicas para el } \\
\text { consumo dentro del establecimiento }\end{array}$ & 137 \\
\hline Expendio a la mesa de comidas preparadas & 144 \\
\hline $\begin{array}{c}\text { Comercio al por menor de productos agrícolas } \\
\text { para el consumo en establecimientos } \\
\text { especializados }\end{array}$ & 147 \\
\hline Transporte de pasajeros & 156 \\
\hline $\begin{array}{l}\text { Comercio de partes, piezas (autopartes) y } \\
\text { accesorios (lujos) para vehículos automotores }\end{array}$ & 169 \\
\hline $\begin{array}{c}\text { Comercio al por menor en establecimientos no } \\
\text { especializados, con surtido compuesto } \\
\text { principalmente }\end{array}$ & 198 \\
\hline $\begin{array}{c}\text { Comercio al por menor de artículos de } \\
\text { ferretería, pinturas y productos de vidrio en } \\
\text { establecimientos }\end{array}$ & 275 \\
\hline $\begin{array}{c}\text { Comercio al por menor de productos } \\
\text { farmacéuticos y medicinales, cosméticos y } \\
\text { artículos de tocador }\end{array}$ & 303 \\
\hline $\begin{array}{c}\text { Comercio al por menor de prendas de vestir y } \\
\text { sus accesorios (incluye artículos de piel) }\end{array}$ & 355 \\
\hline $\begin{array}{c}\text { Comercio al por menor en establecimientos no } \\
\text { especializados con surtido compuesto } \\
\text { principalmente }\end{array}$ & 646 \\
\hline TOTAL & 156 \\
\hline
\end{tabular}

Fuente: Cámara de Comercio de Ocaña 2016

Por consiguiente, Ocaña es un municipio con una alta concentración empresarial con 5.905 Mipymes registradas en Cámara de Comercio y 9.428 personas vinculadas. De acuerdo a las proyecciones del (DANE, 2015), Ocaña cuenta con una población de 98.229 de habitantes, por lo tanto, la actividad empresarial de Ocaña cubre el 9,6\% de la población, es decir 9.428 familias beneficiadas.

Como se observa en la tabla II, el $98 \%$ de las empresas de Ocaña, corresponden a microempresas. Es evidente entonces que el sector microempresarial es el impulsor y dinamizador de la economía ocañera y su área de influencia. Sin embargo, en la actualidad, el municipio carece de una entidad que se encargue de asesorar e impulsar el desarrollo de este sector, de tal manera que pueda incursionar en nuevos mercados tanto a nivel nacional como internacional.

En consecuencia, y considerando la gran cantidad de empresas existentes en el municipio, su diversidad y dinamismo, resulta de vital importancia lograr identificar y unir sinergias que permitan potencializar sus fortalezas como mecanismo facilitador para consolidarse competitivamente en nuevos mercados. Por tal razón, y en la búsqueda de lograr competir en mercados regionales, nacionales e internacionales, la coopetencia es una estrategia competitiva donde la innovación es fundamental para la generación de acciones conjuntas, trabajo en equipo y establecimiento de alianzas, las cuales buscan captar nuevos mercados para mejorar la rentabilidad e incrementar la productividad de cada una de las Mipymes en el municipio.

Por lo anterior, y con el propósito de participar en la transformación del tejido empresarial, social y ambiental del municipio, se plantea como estrategia competitiva desarrollar para las Mipymes de Ocaña el modelo de coopetencia a través de una corporación denominada "Ocaña Ciudad de Negocios" que se sustenta en los conceptos de Desarrollo Sostenible, de Responsabilidad Social Empresarial (RSE) y demás conceptos ya abordados.

El Desarrollo Sostenible, como lo enuncia (Angel, 2004, pág. 104), “(...) aquel tipo de desarrollo que atiende las necesidades del presente sin comprometer generaciones futuras de atender a sus propias necesidades (tomado del informe Brundtland, 1987,8)". Por consiguiente, en lo que compete a Responsabilidad Social Empresarial (RSE), el autor en mención afirma que: "La RSE tiene que ver necesariamente con una forma de interpretar las relaciones entre la empresa y la sociedad (...)" (p. 35).

De igual manera, Bloom y Gundlach (2001), citado por (Cancino \& Morales, 2008, pág. 10) expresaron que:

La RSE guarda relación con la obligación que tiene una empresa con diversos grupos de interés, como los trabajadores, los clientes, las personas y los grupos a quienes pueden afectar las políticas y prácticas corporativas. Parte relevante de gestionar estas obligaciones es intentar minimizar cualquier daño de 
largo plazo que una empresa puede generar sobre la sociedad.

Con base en lo anteriormente expuesto e incorporando las fortalezas identificadas en el diagnóstico situacional de cada una de las Mipymes de Ocaña las cuales conformaron la población y muestra de la investigación, se confirma la necesidad de diseñar el modelo de Coopetencia, el cual se desarrollará a través de una Corporación que agremie a las Mipymes de Ocaña, quienes deberán adoptar el sistema de gestión administrativa, de gestión ambiental empresarial y sus productos se identificarán a través de la marca colectiva Ocaña Ciudad de Negocios como estrategia corporativa, con lo cual se fortalecerán los procesos productivos, coadyuvando al desarrollo económico, ambiental y social del municipio, de tal manera que facilite la integración de las estrategias competitivas propuestas por (Serna, 2014) enunciadas en el artículo tales como "coopetencia, designio estratégico, la estrategia del océano azul y la hipercompetencia" (p.43), teniendo en cuenta que el presente artículo se focaliza en la estrategia de coopetencia.

\section{Metodología}

La investigación se desarrolló bajo el enfoque cuantitativo, en ese sentido, se abordó el tipo de investigación de nivel descriptiva y exploratoria.

Por lo anterior, (Arias, 2004, pp. 21-22) describe que la investigación descriptiva consiste en: “... caracterización de un hecho, fenómeno, o grupo con el fin de establecer su estructura o comportamiento" (p.22). Por tanto ésta parte de la caracterización de las empresas objeto de estudio.

En lo que corresponde a la investigación exploratoria el autor en mención la define como: “... aquella que se efectúa sobre un tema u objeto desconocido o poco estudiado..." (p.21). Para lo cual, en la investigación se abordó un modelo de coopetencia para las Mipymes propuesto para las Mipymes de Ocaña, siendo éste poco estudiado e implementado en el municipio.

Por consiguiente, el diseño de la investigación fue de carácter documental y de campo, teniendo en cuenta que para Arias (2014) las define de la siguiente forma:

Investigación Documental: Es un proceso basado en la búsqueda, recuperación, análisis, crítica e interpretación de datos secundarios, es decir, los obtenidos y registrados por otros investigadores en fuentes documentales: impresas, audiovisuales o electrónicas. Como en toda investigación, el propósito de este diseño es el aporte de nuevos conocimientos. (p.25)

Investigación de Campo: Consiste en la recolección de datos directamente de los sujetos investigados, o de la realidad donde ocurren los hechos (datos primarios), sin manipular o controlar variable alguna. (p.28)
A manera de colofón, como fuente primaria se tomó el paradigma de coopetencia creado en el año 1996 y desarrollado por Barry Nelebuff y Adam Branderburger. En lo referente al diseño de campo se recolectaron los datos directamente de las Mipymes de Ocaña, aplicándose como instrumento de recolección de información la encuesta permitiendo con la tabulación de los resultados evidenciar la realidad de la situación organizacional, ambiental y socioeconómica de las mismas y por ende contribuyó a la descripción del diagnóstico situacional como la parte descriptiva en la definición del manual de gestión administrativa y el de gestión ambiental empresarial.

Por otra parte, en lo referente a la población y muestra objeto de la investigación, se tomó como criterio definirla por las 64 Mipymes quienes participan en la Feria del Arte y Sabor, organizada, liderada y ejecutada por la (Cámara de Comercio de Ocaña, 2015)

Tal como se expresó en lo expuesto en el desarrollo de la investigación de campo, es necesario precisar que las técnicas e instrumentos de recolección de datos aplicados en la investigación, éstos se desarrollaron conforme a los diseños de investigación tanto documental como de campo, así:

Tabla IV. Diseños de investigacion

\begin{tabular}{|c|c|c|}
\hline $\begin{array}{c}\text { Diseño de la } \\
\text { investigación }\end{array}$ & Técnicas & Instrumentos \\
\hline Documental & Análisis Documental & Fichas Bibliográficas \\
\hline \multirow{2}{*}{ De Campo } & $\begin{array}{c}\text { Observación: No } \\
\text { Estructurada } \\
\text { Encuesta: Escrita }\end{array}$ & $\begin{array}{c}\text { Diario de Campo } \\
\text { Cámaras: } \\
\text { Fotográficas y de } \\
\text { video. }\end{array}$ \\
& & Cuestionario \\
\hline
\end{tabular}

\section{Resultados y Discusión}

Diagnóstico situacional Para el desarrollo del diagnóstico situacional de las 64 Mypimes encuestadas en la ciudad de Ocaña se tuvo en cuenta cuatro componentes fundamentales para el logro del modelo de coopetencia: el organizacional, el económico, el de gestión ambiental empresarial y de responsabilidad social, (figura 2 ):

\section{Componente organizacional}

Involucró aspectos propios del proceso administrativo, como la planeación, la organización, el direccionamiento estratégico incorporando los principios corporativos, la visión y la misión de las Mipymes de Ocaña.

En la encuesta se halló que el $60 \%$ de los empresas tienen plenamente definidos los perfiles por cargo, así como las funciones y responsabilidades, del mismo modo, el $80 \%$ utiliza como mecanismo de enganche el contrato laboral. 


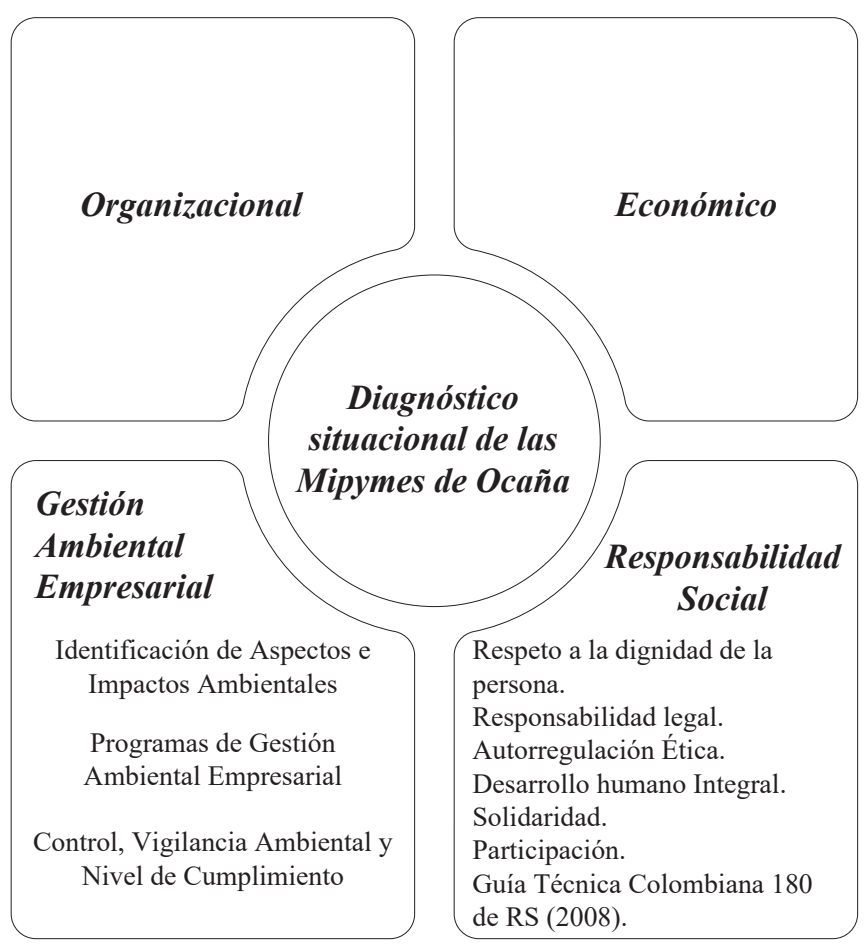

Figura 2. Coopetencia: cooperacion + competencia

Uno de los aspectos relevantes encontrados en los resultados de la encuesta está expresado en que el $64 \%$ de las organizaciones tienen claro y definido su misión y visión, así como sus valores y principios corporativos; el $72 \%$ cuenta con un organigrama.

En cuanto a la planeación y organización, se encontró que el $72 \%$ identifican fortalezas y debilidades, oportunidades y amenazas, lo cual el $60 \%$ lo utilizan de insumo para diseñar planes de acción e implementar estrategias para alcanzar las metas propuestas.

En lo referente a servicio al cliente, el $64 \%$ de las organizaciones encuestadas respondieron que cuentan con políticas y procedimientos de atención al cliente; además, el 44\% implementa políticas de devolución y ventas; el 56\% utiliza el sondeo para medir el grado de satisfacción del cliente.

\section{Componente económico}

La encuesta se encaminó a explorar sobre la manera como las Mipymes utilizan sus recursos financieros durante el proceso de producción de bienes y servicios.

En la indagación se encontró que de las 64 Mipymes encuestadas el $84 \%$ de ellas tienen calculado sus costos de producción; solamente el $48 \%$ se han preocupado en proyectar sus ingresos por concepto de ventas; y el $64 \%$ tiene calculado su punto de equilibrio, lo cual le permite al propietario tener definido el volumen mínimo de ventas para evitar pérdidas operacionales.
Ante la pregunta del número de empleados, el $96 \%$ de los encuestados respondieron tener entre 1 y 10 trabajadores, característica relevante que clasifica a estas organizaciones como microempresas (Ley 905 de 2004) y fuente importante en la generación de empleo en el mercado laboral del municipio de Ocaña.

El 52\% de los bienes producidos cuentan con su respectiva ficha técnica y empaque; el $80 \%$ tiene plenamente identificado el mercado meta, para lo cual el $52 \%$ utiliza portafolio de productos; el $72 \%$ tiene una marca registrada, utilizada por el $64 \%$ en estrategias de publicidad; el $74 \%$ utiliza como canales de comercialización las ventas directas y los puntos de venta, y el $23 \%$ a las comercializadoras.

Solamente el $32 \%$ llevan contabilidad; el 52\% cumplen con sus obligaciones tributarias y el $32 \%$ de las mipymes encuestadas generan documentos equivalentes a la factura de venta.

\section{Componente ambiental}

Se evaluaron aspectos e impactos ambientales involucrados en la operación de cada Mipyme de Ocaña, entendiendo esto como la base de planificación de un Sistema de Gestión Ambiental (SGA) que garantice el uso responsable y racional de los recursos involucrados en cada proceso de la pequeña industria.

Uno de los principales indicadores para la gestión ambiental es la ubicación de las Mipymes en sectores residenciales, siendo esto un común denominador con el $94 \%$, lo que dificulta la evaluación de aspectos e impactos ambientales, reflejando también una baja demanda de bienes y servicios ambientales, apropiados a características de cada unidad de negocio.

Otro de los factores influyentes en el estudio es la identificación de requisitos legales ambientales aplicables a la operación de las Mipymes, donde el $98 \%$ de los encuestados aseguraron no tener conocimiento sobre dichos requisitos, su identificación, aplicación y acercamiento a las autoridades ambientales correspondientes, lo que traduce un total desconocimiento de la norma y problemas con la regulación ambiental.

En cuanto a otras conductas ambientales, el $65 \%$ reconoció que tiene $\mathrm{o}$ ha adquirido conocimiento para correctos procedimientos de reciclaje, mientras que un $45 \%$ aceptó que realizaba separación en la fuente, en donde la caracterización de residuos fue la siguiente: Realiza separación de plásticos, cartón y vidrios $43 \%$, reconoce que estos materiales son los mayormente aprovechables $20 \%$, realiza transformación de subproductos o residuos de la materia prima $5 \%$, reutiliza o ahorra agua $65 \%$ y ahorra energía $75 \%$.

Así mismo se tomó como referencia lo estipulado por la (Guía Técnica Colombiana 180 de RS, 2008) promulgada a través del Instituto Colombiano de Normas Técnicas (ICONTEC), en el 
literal de la norma 3.2 donde señala los principios de responsabilidad social: Respeto a la dignidad de la persona, Responsabilidad legal, Autorregulación Ética, Participación, Solidaridad y Desarrollo Humano Integral.

La situación de las Mipymes en el contexto municipal y el rol que se desempeña dentro de cada una de ellas, lleva a que se piense de manera primordial la sinergia que se da entre el empleador, el empleado y su relación estrecha con el entorno, para lo cual la encuesta arrojó los siguientes resultados:

La prioridad en la operación de negocio es la integridad física del empleado, por lo tanto el $65 \%$ mantiene estándares de gestión corporativa, el $85 \%$ con valores como la ética y la honestidad en su visión de negocio, el 10\% mantiene una relación clara y verificable con sus vecinos en el área de influencia de su operación, el 25\% involucra conductas amigables con el medio ambiente en relación con su operación de negocio, el 34\% toma decisiones en su Mipyme basado en preceptos de derechos humanos. Con los empleados los salarios son acordes a estándares nacionales, con salarios dignos 15\%, igualmente apoya iniciativas sociales que reconstruyan el tejido humano en el área de influencia de su operación $5 \%$, y el 7\% mantiene una relación con sus pares en Mipymes para incorporar estrategias de compensación y responsabilidad donde se concentra su operación.

Con todo lo anteriormente expuesto se vislumbra la necesidad de enfocar y aunar esfuerzos que viabilicen el desarrollo de la coopetencia como modelo de gestión empresarial en las Mipymes de Ocaña a través de acciones conjuntas donde cada una de ellas obtengan beneficios particulares, comunes y con mayores rendimientos que no obtendrán si actúan de forma independiente en el mercado. Por ello se conformó una Corporación, con la cual se fortalecerán los procesos administrativos, orientados a la cultura organizacional y con perspectiva hacia la responsabilidad social y a la gestión ambiental empresarial con factores diferenciadores que les permitan ser sustentables, productivos y competitivos en los diferentes mercados.

\section{Componente de responsabilidad social}

Definido según la Comisión Europea (2001), citado por (Cancino \& Morales, 2008, pág. 11) "la RSE es un concepto en el cual las áreas productivas integran, de una manera voluntaria, consideraciones sociales y ambientales en sus operaciones y en las relaciones con sus grupos de interés".

Teniendo en cuenta, que la investigación fue orientada a incentivar en las Mipymes Ocañeras la estrategia competitiva (coopetencia) propuesto por Serna (2014) y como se expresó en el párrafo anterior es un modelo propuesto por (Nelebuff \& Brandenburger, 1996), se logró consolidar la corporación Ocaña Ciudad de Negocios encargada de promover e impulsar a sus asociados a consolidarse en el sistema económico de una manera sostenible con proyección hacia la inserción a la dinámica de la globalización económica, con el apoyo de alianzas estratégicas con instituciones como: la Universidad Francisco de Paula Santander Ocaña, Servicio Nacional de Aprendizaje SENA - Ocaña y la Cámara de Comercio de Ocaña.

Mediante las alianzas estratégicas se busca consolidar sinergias que faciliten el acompañamiento, seguimiento y evaluación de las diferentes estrategias que se implementen para la mejora continua de cada una de las organizaciones pertenecientes a la Corporación Ocaña Ciudad de Negocios.

Asimismo, se propenderá por mejorar las condiciones empresariales de los asociados, promover el desarrollo sostenible del municipio, formular proyectos de inversión y participar en eventos nacionales e internacionales.

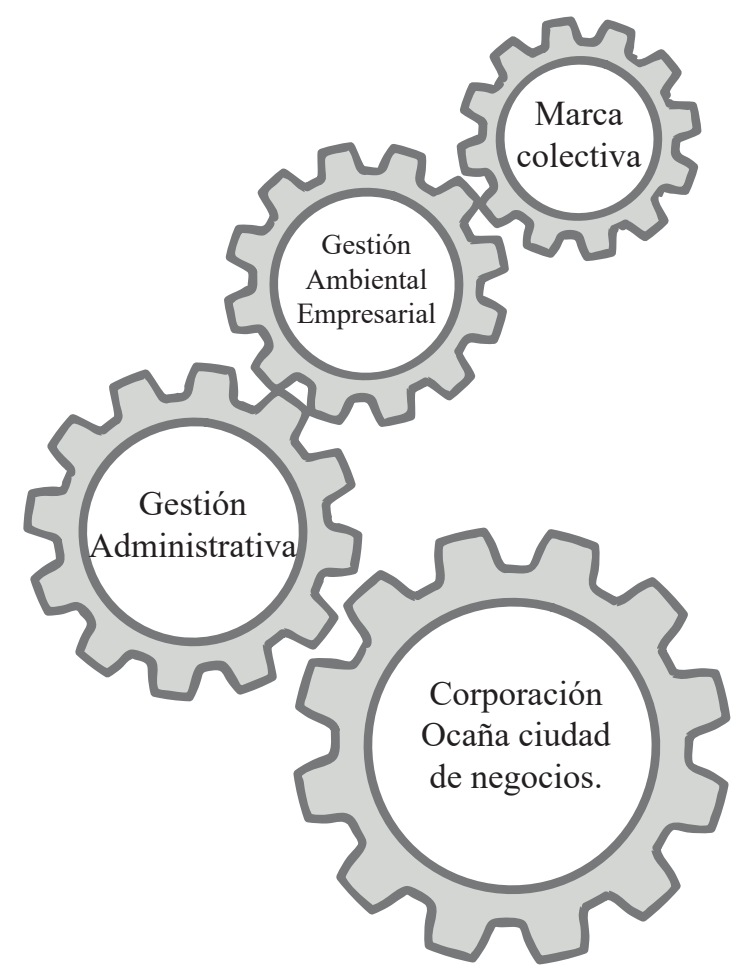

Figura 3. Modelo de Coopetencia para las Mipymes de Ocaña

En la figura 3 se muestra la articulación del modelo de coopetencia a desarrollarse en la Corporación Ocaña Ciudad de Negocios, teniendo en cuenta los cuatro componentes fundamentales para el logro del modelo de coopetencia: el organizacional, el económico, el de gestión ambiental empresarial y de responsabilidad social pensando en la competencia más la cooperación (coopetencia), el análisis de los componentes de la encuesta demostraron la necesidad de la inclusión de formas diferenciadoras para observar las Mipymes, donde se realza el factor humano y su relación con el entorno, el cuidado por el medio ambiente y el a acercamiento a los derechos humanos como estrategia de desarrollo sostenible, que pueda impulsar la economía municipal a través de 
una corporación desde la mirada de Ocaña como ciudad de negocios, innovadora, inteligente, emprendedora, social y disminuyendo una huella ecológica que por la operación sin planificación amenaza los recursos naturales y la dignidad laboral.

Así mismo, quienes hagan parte de la Corporación Ocaña Ciudad de Negocios, implementarán el sistema de gestión administrativa, de gestión ambiental empresarial, y como estrategia corporativa incorporarán en su publicidad, empaques y etiquetas la marca colectiva Ocaña Ciudad de Negocios, (figura 4).

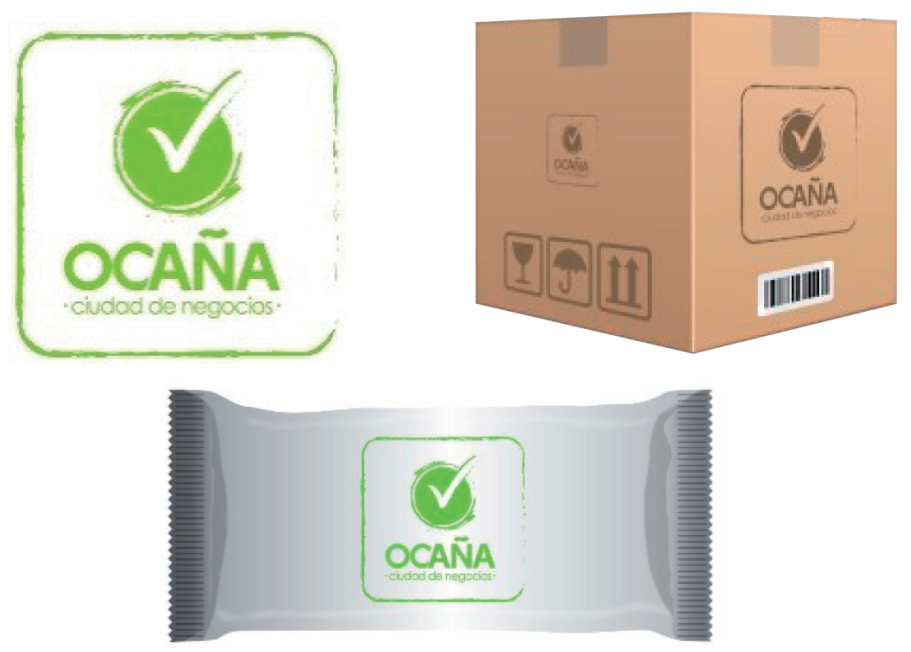

Figura 4. Publicidad, empaques y etiquetas de la marca colectiva Ocaña ciudad de negocios

Fuente: Oficina de Multimedios UFPS Ocaña

Es de resaltar que la marca colectiva fue producto del consenso realizado con los micropempresarios que hicieron parte de la muestra, donde se evaluaron factores como: la competencia, la innovación, la imagen corporativa, el impacto publicitario y el impacto ambiental.

\section{Financiamiento}

El presente artículo es producto de la investigación denominada Coopetencia Empresarial para las Mipymes y empresas sin ánimo de lucro de Ocaña y su Provincia, Norte de Santander, desarrollada por el Grupo de Investigación ROTÃ del cual hacen parte los autores. La investigación principal fue presentada en la Convocatoria No. 02 de la División de Investigación y Extensión- DIE, avalada y financiada por la Universidad Francisco de Paula Santander Ocaña.

\section{Agradecimientos}

Se agradece de manera muy especial al Ingeniero Ambiental Luis Orlando Vergel Granados y al Especialista Carlo
Fernando D’Vera Pérez por sus aportes y experiencia, de igual manera se resalta al Investigador Carlo Fernando D'Vera Pérez quien hizo parte de la realización de la investigación.

\section{Referencias}

[1] COPI. (2016). acopi. org. Recuperado en: http://acopi.org.co/quienes-somos/

[2] Angel, J. L. (2004). Responsabilidad Social y los principios del Desarrollo Sostenible como fundamentos teóricos de la información social de la empresa. Madrid: Esic Editorial, 104. Arias, G. (2004). EL proyecto de investigación. Introducción a la metodología. Caracas: Episteme c.a, 21-22-25-28.

[3] Cámara de Comercio de Ocaña. (01 de 04 de 2015). Boletines cámara de Comercio de Ocaña. Recuperado el 07 de Octubre de 2015, en: http://www.camaraocana.com/CMS/archivos/boletin/Abril.pdf

[4] Cancino, C., \& Morales, M. (2008). Responsabilidad Social Empresarial. Serie Documento Docente(1), 9-58.

DANE. (30 de 06 de 2015). Demografía y Población DANE. Recuperado el 22 de Octubre de 2015, de Proyecciones de población: http://www.dane.gov.co/index.php/poblacion-y-demografia/proyecciones-de-poblacion

[5] Guía Técnica Colombiana 180 de RS. (2008). Recuperado el 16 de Abril de 2016, de Icontec: file://C:/Users/usuario/Downloads/GTC180\%20(1).pdf

[6] Jaramillo, C. (2005). Compettividad. Abya Yala, 253.

LaNotaEconómica.co. (19 de 10 de 2010). El mundo de las Pymes. La Nota Económica.co, s/p.

[7] Ley 905. (2004). Recuperado el 20 de Enero de 2015, de Alcaldía de Bogotá: http://www.alcaldiabogota.gov.co/sisjur/normas/Norma1.jsp?i=14501

[8] Nelebuff, B. J., \& Brandenburger, A. M. (1996). Co-opetition (Grupo Editorial Norma ed.). (J. Cárdenas Nannetti, Trad.) New York: Currency Books Doubleday, 28.

[9] Serna, H. (2014). Gerencia Estratégica: Teoría, metodología, mapas estratégicos, indices de gestión, alineamiento, ejecución estratégica (11 ed.). Bogotá: Panamericana, 32-33-43.

[10] Vega, L. (2004). El Estado como Sistema: el enfoque sistémico y evaluación de las políticas públicas. El caso de la política en la formulación, seguimiento ambiental. Bogotá: Ed. Systemical ltda, 104. 